\title{
Thermoelectric properties of FeAs based superconductors, with thick perovskite- and Sm-O fluorite-type blocking layers
}

\author{
S. J. Singh ${ }^{\mathrm{a}, \mathrm{b}}$, J. Shimoyama ${ }^{\mathrm{a}}$, H. Ogino ${ }^{\mathrm{a}}$ and K. Kishio ${ }^{\mathrm{a}}$ \\ ${ }^{a}$ Department of Applied Chemistry, The University of Tokyo, 7-3-1 Hongo, Bunkyo-ku, Tokyo \\ 113-8656 Japan \\ ${ }^{b}$ Leibniz-Institute for Solid State and Material Research, IFW-Dresden, D-01171 Dresden \\ Germany
}

${ }^{*}$ Corresponding author.

E-mail address: s.j.singh@ifw-dresden.de

Tel.:+49-0-351-4659-157

Fax: +49-0-351-4659-313. 


\begin{abstract}
The transport properties (electrical resistivity, Hall and Seebeck coefficient, and thermal conductivity) of iron based superconductors with thick perovskite-type oxide blocking layers and fluorine-doped SmFeAsO were studied to explore their possible potential for thermoelectric applications. The thermal conductivity of former compounds depicts the dominated role of phonon and its value decreases rapidly below the $T_{\mathrm{c}}$, suggesting the addition of scattering of phonons. Both the Seebeck coefficient $(S)$ and Hall coefficient $\left(R_{\mathrm{H}}\right)$ of all samples were negative in the whole temperature region below $300 \mathrm{~K}$, indicating that the major contribution to the normal state conductivity is by electrons. In addition, the profile of $S(T)$ and $R_{\mathrm{H}}(T)$ of all samples have similar behaviours as would be expected for a multi-band superconductors. Although the estimated thermoelectric figure of merit $(Z T)$ of these compounds was much lower than that of practically applicable thermoelectric materials, however its improvement can be expected by optimizing microstructure of the polycrystalline materials, such as densification and grain orientation.
\end{abstract}

\title{
Keywords:
}

FeAs based superconductors

Thermal conductivity

Hall effect

Thermo-electric power 


\section{Introduction}

Iron-arsenic based high temperature superconductors have a large number of materials with significant differences in their chemical compositions [1-2]. Their physical properties can be tuned by both elemental substitutions and external pressure. So far, the superconducting transition temperature, $T_{\mathrm{c}}$, has been raised up to $\sim 58 \mathrm{~K}$ for REFeAsO (RE1111, RE $=$ rare earth) system [3-5] by F doping at O-site. Among these superconducting materials, a series of iron pnictides composed of thick perovskite-type oxide blocking layers have been also found [6-10]. Several structure types and various combination of constituent elements have been reported due to the flexibility of the perovskite-type structure in such compounds. Furthermore, this system shows relatively high $T_{\mathrm{c}}$ over $40 \mathrm{~K}$, for example; $T_{\mathrm{c}}$ is $47 \mathrm{~K}$ for $\left(\mathrm{Fe}_{2} \mathrm{As}_{2}\right)\left(\mathrm{Ca}_{4}\left(\mathrm{Mg}_{0.25} \mathrm{Ti}_{0.75}\right)_{3} \mathrm{O}_{8}\right)$ (22438) [8] and $41 \mathrm{~K}$ for $\left(\mathrm{Fe}_{2} \mathrm{As}_{2}\right)\left(\mathrm{Ca}_{5}\left(\mathrm{Sc}_{0.5} \mathrm{Ti}_{0.5}\right)_{4} \mathrm{O}_{11}\right)(2254 \underline{11})$ [9].

Searching good thermoelectric materials are a challenging issue from physics and device application point of view. Many of the iron based superconductors have been reported for their large thermoelectric power at low temperatures above $T_{\mathrm{c}}$ [11-14]. It is an important material parameter along with the electrical and thermal conductivity that determines the efficiency of the thermoelectric applications. The efficiency of thermoelectric device increases monotonically with the dimensionless figure-of-merit $Z T\left(=\left(S^{2} / \rho \kappa\right) T\right)$, where $S$ is the thermoelectric power or Seebeck coefficient, $\rho$ and $\kappa$ are the electronic resistivity and thermal conductivity, respectively. For a thermoelectric application, a large $|S|$ with low $\rho$ and low $\kappa$ are required. It has been reported that Bi-Sb alloy, generally, show the best thermoelectric performance and a thermoelectric figure of merit, $Z T$ of 0.5 in the low temperature range $\sim 80-100 \mathrm{~K}[15-16]$. The behaviour and magnitude of thermoelectric power of the iron pnictide superconductors exhibit approximately similar to the reported one that is obtained in Bi-Sb alloys [15]. The large value of 
thermoelectric power of this new family could be possible because of the electron correlations or to 2D electron confinement in FeAs layers but its origin is still not clear. This large thermoelectric power can make it a promising candidate for the application around liquid nitrogen temperature, $77 \mathrm{~K}$. More than 150 compounds have been developed as iron based superconductors with different blocking layers [17]. Generally, layered materials have been actively studied because of their low dimensional electronic states and could result in high thermoelectric properties as realized in $\mathrm{Bi}_{2} \mathrm{Te}_{3}$ [18], cobalt oxides [19] and $\mathrm{CsBi}_{4} \mathrm{Te}_{6}$ [20], $\mathrm{BiS}_{2}$ based compounds $\mathrm{LaOBiS}_{2}$ [21]. Hence, it will be interesting to investigate and compare the thermal properties of iron based superconductors belonging to different categories with different types of blocking layer. Furthermore, the dependence of thermoelectric power of Sm1111 with different F-doping levels should also be interesting to study. With these motivations, we have chosen two categories of iron based superconductors having different blocking layers: one is Sm based oxypnictide superconductors with SmO blocking layer belonging to RE1111 which generally gives the highest transition temperature by F-doping and other belongs to iron based superconductor with thick perovskite-like oxide blocking layers. The thermoelectric properties of Sm1111 have been reported by some groups $[11,14]$ where the samples were synthesized at a high temperature $\left(\sim 1150^{\circ} \mathrm{C}\right)$, however to the best of our knowledge, the thermal properties of iron based superconductors with thick perovskite-type oxide blocking layers especially 22438 , 225411 have not been reported.

In this paper, we have studied the temperature dependence of electrical resistivity $\rho(T)$, thermoelectric power $S(T)$, thermal conductivity $\kappa(T)$, Hall effect $R_{\mathrm{H}}(T)$ and the calculated figure of merit $(Z T)$ of the two iron based superconductors with thick perovskite blocking layers $\left(\mathrm{Fe}_{2} \mathrm{As}_{2}\right)\left(\mathrm{Ca}_{4}\left(\mathrm{Mg}_{0.25} \mathrm{Ti}_{0.75}\right)_{3} \mathrm{O}_{8}\right) \quad$ (22438) and $\left(\mathrm{Fe}_{2} \mathrm{As}_{2}\right)\left(\mathrm{Ca}_{5}\left(\mathrm{Sc}_{0.5} \mathrm{Ti}_{0.5}\right)_{4} \mathrm{O}_{11}\right) \quad$ (22541ㅣ), and 
$\operatorname{SmFeAs}\left(\mathrm{O}_{1-x} \mathrm{~F}_{x}\right)(\mathrm{Sm} 1111)$ which are prepared by low temperature $\left(\sim 900^{\circ} \mathrm{C}\right)$ synthesis method [4] and has the highest $T_{\mathrm{c}}(\sim 58 \mathrm{~K})$ among the iron pnictide superconductors. All thermal coefficients and electrical resistivity of iron based superconductors with thick perovskite-type oxide layers are discussed and depicts approximately the similar behaviour with Sm1111. The temperature dependence of thermoelectric power and Hall coefficient of 22438 and $2254 \underline{11}$ can be explained by considering the multiband nature in which electron type charge carriers are playing the dominant role as in the case of oxypnictide superconductors.

\section{Experimental}

Sintered bulk samples with nominal compositions of $\left(\mathrm{Fe}_{2} \mathrm{As}_{2}\right)\left(\mathrm{Ca}_{4}\left(\mathrm{Mg}_{0.25} \mathrm{Ti}_{0.75}\right)_{3} \mathrm{O}_{8}\right)$ (22438) and $\left(\mathrm{Fe}_{2} \mathrm{As}_{2}\right)\left(\mathrm{Ca}_{5}\left(\mathrm{Sc}_{0.5} \mathrm{Ti}_{0.5}\right)_{4} \mathrm{O}_{11}\right)$ (225411) were synthesized by single step solid state reaction starting from $\mathrm{CaO}, \mathrm{Ti}, \mathrm{FeAs}, \mathrm{TiO}_{2}, \mathrm{Sc}_{2} \mathrm{O}_{3}$ and $\mathrm{MgO}$ as described previously [8-9, 22]. Bulk $\mathrm{SmFeAsO}_{0.88} \mathrm{~F}_{0.12}$ and $\mathrm{SmFeAsO}_{0.8} \mathrm{~F}_{0.2}$ samples were synthesized by low temperature synthesis route and the use of starting materials of $\mathrm{SmAs}, \mathrm{FeF}_{2}, \mathrm{Fe}_{2} \mathrm{O}_{3}$ and $\mathrm{FeF}_{2}$, as details reported in [4-5]. The resulting samples were characterized by powder X-ray diffraction (XRD) with $\mathrm{Cu}-K_{\alpha}$ radiation and microstructural analysis as discussed in our previous paper [22].

Transport measurements were carried out with Physical Properties Measurements System (Quantum Design PPMS Model 6000) in the temperature range 2-300 K and in field up to $9 \mathrm{~T}$. Hall coefficient $\left(R_{\mathrm{H}}\right)$ were determined by measuring the transverse resistivity at selected temperatures sweeping the field from -9 to 9 T. The Seebeck coefficient $(S)$ and thermal conductivity $(\kappa)$ were measured using a thermal transport option in continuous scanning mode with slow heating rate. For the evaluation of thermoelectric properties, the samples of several $\mathrm{mm}$ in length were cut in the shape of parallelepipeds with a heat flow along the longest axis. 
The error for these measurements is estimated $\sim 10 \%$ near the room temperature and $\sim 5 \%$ below $200 \mathrm{~K}$ due to the radiation losses. However, it becomes negligible at lower temperatures.

\section{Results and discussions}

The XRD patterns for 22438 and 225411 samples showed that each samples formed as a main phase with small amount of impurities as discussed in [22]. The crystal structures of Sm1111 and 22438, 225411 are shown in Fig. 1. The lattice constants of the samples of 22438, 225411, $\mathrm{SmFeAsO}_{0.88} \mathrm{~F}_{0.12}$ and $\mathrm{SmFeAsO}_{0.8} \mathrm{~F}_{0.2}$ were evaluated as $a=3.873(1) \AA$ and $c=$

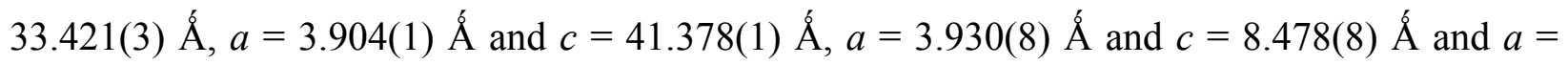

3.924(1) $\AA$ and $c=8.487(4) \AA$, respectively. The samples of 22438 and $2254 \underline{11}$ were porous with low relative densities of $43 \%$ and $41 \%$, respectively, estimated by assuming their theoretical densities as 4.435 and $4.355 \mathrm{~g} / \mathrm{cm}^{3}$ respectively, while the relative density of Sm1111 samples were approximately $50 \%[5]$.

Fig. 2(a) shows the temperature dependence of resistivity under zero magnetic field up to $300 \mathrm{~K}$ for these four samples. All samples exhibited superconducting transitions with the $T_{\mathrm{c}}$ (onset) of approximately 48, 41, 51 and $57.2 \mathrm{~K}$ for 22438, 225411, $\mathrm{SmFeAsO}_{0.88} \mathrm{~F}_{0.12}$ and $\mathrm{SmFeAsO} \mathrm{.}_{0.8} \mathrm{~F}_{0.2}$, respectively [22]. Although, all these samples showed metallic behaviour in the normal state, the resistivity value is higher for $2254 \underline{11}$ and 22438 compared to Sm1111 samples that might be due to enhanced weak coupling characteristics of iron based superconductor by thick perovskite-type blocking layer as discussed elsewhere [22].

To confirm the dominant conduction mechanism, we have investigated the temperature dependence of thermoelectric power $(S)$ for these four samples as shown in Fig. 2(b). For Sm1111 samples, the absolute value of thermoelectric power $(|S|)$ increases with decreasing 
temperature, while at low temperatures it develops a broad maximum below $100 \mathrm{~K}$. After that $|S|$ decreases rapidly and becomes zero around $T_{\mathrm{c}}{ }^{\text {(onset) }}$. This behaviour is well coincided with a former report studied for Sm1111 with $x=0.075$ and 0.15 [11] synthesized by high temperature synthesis route $\left(\sim 1200^{\circ} \mathrm{C}\right)$. The unusual large absolute value of $S$ has also observed in various 1111-type compounds [11-14]. Although, it is more substantial to discuss the relationship between the actual fluorine concentration and maximum value of $|S|$, a monotonic dependencies of $|S|_{\max }$ on nominal fluorine concentration and $T_{\mathrm{c}}$ were confirmed as shown in Fig. 3(a) and 3(b). It indicates that $|S|_{\max }$ does not depend on the synthesis route and reduces with increasing Fcontent which might be possible due to the enhancement of impurity phase or weak grain connectivity at higher F-doping [4-5]. For 22438 and 2254111, the profile of $S(T)$ curves are similar to those of Sm1111 samples, while $|S|$ has much smaller value $(-25 \mu \mathrm{V} / \mathrm{K})$ as similar to $\mathrm{FeTe}_{1-x} \mathrm{Se}_{x}$ [11]. The thermoelectric power is negative in the whole temperature range above $T_{\mathrm{c}}$, suggesting the electron type charge carriers are playing a major role in these compounds. The observed $S(T)$ behaviours for 22438 and 225411 could be explained by considering a multiband scenario of the electronic state in these compounds as in the other FeAs-based superconductors, where both electron and hole pockets are possible for the electrical transport [23-24]. The thermoelectric power is then a mixture of the individual thermoelectric power of each electron and hole bands. On this basis, the decrease in the thermoelectric power can be understood by considering the contribution of an electron or a hole band to the total $S$, or a change in charge carrier scattering mechanism. We also note that the $|S|_{\max }$ appeared at lower temperature for 22438 and 225411 than those of Sm1111 samples.

The temperature dependence of thermal conductivity of these four samples is displayed in Fig. 2(c). Actually heat is transported in solid materials by both lattice vibration waves (phonons) 
and free electrons and generally, thermal conductivity is the sum of these two contributions. The thermal conductivity of Sm1111 samples is almost constant from room temperature to transition temperature. Invoking the Wiedemann-Franz law $\left(\kappa_{\mathrm{e}}=L_{0} T / \rho\right.$, where $\left.L_{0}=2.45 \times 10^{-8} \mathrm{~W} \Omega / \mathrm{K}\right)$ and the measured electrical resistivity $(\rho)$, we estimated the electronic contribution to the thermal conductivity in the vicinity of the transition temperature (at $60 \mathrm{~K}$ ) to be $\sim 60 \%$ of the total heat conduction for $\mathrm{SmFeAsO}_{0.8} \mathrm{~F}_{0.2}$ whereas underdoped $\mathrm{Sm} 1111\left(\mathrm{SmFeAsO}_{0.88} \mathrm{~F}_{0.12}\right)$ has the electronic contribution of $\sim 20 \%$ at $60 \mathrm{~K}$. Below $T_{\mathfrak{c}}$, it shows a pronounced peak similar to other iron based superconductors [11-12, 25-26], which reflects the enhancement of the quasi particle mean free path in the superconducting state. Actually, in the superconducting state, quasi particles condense into cooper pairs and their number decrease rapidly below $T_{\mathrm{c}}$. This in turn gives rise to a reduction in the scattering cross section of quasi particles, and hence the mean free path increases below $T_{\mathrm{c}}$. On the other hand, the thermal conductivity of the samples having thick perovskite-type blocking layer did not show peaks below $T_{\mathfrak{c}}$, while they show nearly same absolute values of $\kappa$ and temperature dependence with Sm1111 samples above $T_{\mathrm{c}}$. Absence of peak below $T_{\mathrm{c}}$ may suggest that both 22438 and 225411 samples have a relatively large distribution of $T_{\mathrm{c}}$ possibly due to their inhomogeneous chemical compositions [22]. The estimated electronic contribution is $\sim 14 \%$ for 22438 and $\sim 4 \%$ for $2254 \underline{11}$ at room temperature, and reached up about $9 \%$ for 22438 and $2 \%$ for 225411 at near $T_{\mathrm{c}}$, respectively. It suggests that in these compounds thermal conductivity is dominated by phonons [25-26]. The thermal conductivity of these iron based superconductors was relatively low as similar to other reports $[11-12,26]$, possibly due to the large scattering rate which can be originated due to weak granular behaviour of iron based superconductors. The weak coupling or porosity may have a 
dramatic influence on thermal conductivity; increasing the pore volume will result in a reduction of the thermal conductivity.

The temperature dependence of $\kappa$ in the low temperature region at $\mu_{0} H=0$ and $9 \mathrm{~T}$ are shown in Fig. 2(d). There is no change in $\kappa$ by the application of magnetic field in 22438. It might be possible due to its extremely steep increase in high upper critical field below $T_{\mathrm{c}}$ as reported elsewhere [22]. On the other hand, $\kappa$ value decreases in the normal state for lightly $\mathrm{F}$ doped Sm1111 whereas it is constant for optimally F doped Sm1111. However, the maximum $\kappa$ remains well evident around $30 \mathrm{~K}$ for both $\mathrm{Sm} 1111$ in a magnetic field of $9 \mathrm{~T}$ below $T_{\mathrm{c}}$. The reduction of $\kappa$ by the application of magnetic field is expected to be due to the decrease in the mean free path of quasiparticles. The magnetic field of $9 \mathrm{~T}$ does not remove the anomaly in Sm1111 which indicates that such a field is much lower than the upper critical field in these samples.

The temperature dependence of Hall coefficient $\left(R_{H}\right)$ for 22438, 225411, $\mathrm{SmFeAsO}_{0.88} \mathrm{~F}_{0.12}$ and $\mathrm{SmFeAsO}_{0.8} \mathrm{~F}_{0.2}$ samples has been studied and is shown in Fig. 4. The inset of Fig. 4 depicts the Hall resistivity $\left(\rho_{\mathrm{xy}}\right)$ with respect to magnetic field from 0 to $9 \mathrm{~T}$ measured for 22438 at various temperatures. It is clear that $\rho_{\mathrm{xy}}$ exhibits a linear relation with respect to magnetic field and is negative at all temperatures above $T_{\mathrm{c}}$. From this set of data, the Hall coefficient $R_{\mathrm{H}}=\rho_{\mathrm{xy}} / H$ was determined and is summarized in Fig. 4 . The negative $R_{\mathrm{H}}$ in the entire temperature range suggests that the superconductivity in all these samples is dominated by electron type charge carriers, which is consistent with the discussion of their thermoelectric power. The absolute values of $R_{\mathrm{H}}$ monotonously increase with a decrease in temperature for all compounds. The temperature dependence of $R_{\mathrm{H}}$ for 22438 and $2254 \underline{11}$ phase becomes larger below $150 \mathrm{~K}$, while Sm1111 samples showed almost constant temperature dependence of $R_{\mathrm{H}}$. It 
is well known that $R_{\mathrm{H}}$ of the conventional metals is nearly independent with respect to the varying temperature. However, the observed Hall coefficient shows a strong temperaturedependent behaviour which cannot described by one single-band model, suggesting the multiband nature [7, 27-29] of these samples. Since the iron based superconductors have the complicated band structure, the interpretation of Hall coefficient behaviours is not so simple. Therefore, $R_{\mathrm{H}}$ provides a qualitative indication of the dominated charge carrier.

To be useful thermoelectric devices, a material must have a large dimensionless figure of merit, $Z T$, which is evaluated by using the data of the electrical resistivity, Seebeck coefficient and thermal conductivity. The calculated $Z T$ of these four samples are shown in Fig. 5. The $Z T$ value increased with lowering temperature for Sm1111 and 22438, and reached to a maxima around $70 \mathrm{~K}$, whereas there is almost no change in $Z T$ values for $2254 \underline{11}$ down to $70 \mathrm{~K}$. Slightly high $Z T$ value of Sm1111 might be due to the relatively high Seebeck coefficient and low resistivity, however, they are still insufficient for applicable level. In general, the $Z T$ value is expected to be greater than 1 as in the case of bismuth antimony telluride and few other bismuth antimony alloy $[18,15,30]$. In the present study, the materials with thick perovskite-type oxide blocking layers exhibit significantly the reduced Seebeck coefficients and the enhanced resistivity coefficient, however, the total thermal conductivity has almost the same values, suggesting the compensation between the electron thermal conductivity and the phonon thermal conductivity. Our results clearly suggest that a role of additional blocking layers in iron based superconductors negatively works on the thermal efficiency of the materials. Moreover, the thermoelectric power is also reduced from under doped $(x \leq 0.12)$ to optimal $(x=0.2)$ F-doped region in Sm1111 whereas the figure of merit is not much affected. While the value of thermoelectric power and the low thermal conductivity are encouraging, particularly for Sm1111 
as reported by other groups also $[11,14]$. The figure of merit improvements may come through better sample morphologies and larger crystalline sizes and via judicious doping processes. By controlling the microstructure such as grain alignment and densification, and the synthesis of dense bulk samples can also be helpful for the enhancement of the power factor $\left(S^{2} / \rho\right)$ as well as the figure of merit, $Z T$.

\section{Conclusions}

We have studied the thermoelectric properties of 22438 and 225411 with respect to Sm1111. It was found that the partial F substitution in Sm1111 is not effective for enhancing figure of merit. The sample 22438 and 225411 showed the higher resistivity value compared to that of Sm1111 whereas both thermoelectric power and thermal conductivity are smaller as an indication of the enhancement of the quasi particle mean free path. The calculated $Z T$ showed the maximum value around $70 \mathrm{~K}$ and this value is comparatively higher for Sm1111. However, all thermal coefficient measurements of 22438 and 225411 have followed the same behaviour similar to Sm1111. In addition, the values of thermoelectric power $|S|$ are approximately same at room temperature and have always negative sign down to $T_{\mathrm{c}}$ for all samples, however, the $|S|$ values are apparently smaller for 22438 and $2254 \underline{11}$ at low temperature. The temperature dependences of the thermoelectric power and Hall coefficient suggest the multiband nature of these perovskite based superconductors and showed the dominant role of electron type charge carriers. Summarily, our results suggest that the addition of thick perovskite-type oxide blocking layers or the higher F substitution in FeAs based superconductors have a detrimental effect on the thermal efficiency of the materials. 


\section{Acknowledgements}

SJS is supported by Japan Society for the Promotion of Science (JSPS), Government of

Japan. This work was partially supported by Japan Science and Technology Agency (JST), PRESTO, SICORP and TRIP. 


\section{References}

[1] Y. Kamihara, T. Watanabe, M. Hirano, H. Hosono, J. Am. Chem. Soc. 130 (2008) 3296.

[2] G. R. Stewart, Rev. Mod. Phys. 83 (2011) 1589.

[3] Z. A. Ren, G. C. Che, X. L. Dong, J. Yang, W. Lu, W. Yi, X. L. Shen, Z.-C. Li, L.-L. Sun, F. Zhou, Z. X. Zhao, Europhys. Lett. 83 (2008) 17002.

[4] S. J. Singh, J. Shimoyama, A. Yamamoto, H. Ogino, K. Kishio, IEEE Trans. Appl. Supercond. 23 (2013) 7300605.

[5] S. J. Singh, J. Shimoyama, A. Yamamoto, H. Ogino, K. Kishio, Supercond. Sci. Technol. 26 (2013) 065006.

[6] H. Ogino, Y. Matsumura, Y. Katsura, K. Ushiyama, S. Horii, K. Kishio, J. Shimoyama, Supercond. Sci. Technol. 22 (2009) 075008.

[7] X. Zhu, F. Han, G. Mu, P. Cheng, B. Shen, B. Zeng, H.-H. Wen, Phys. Rev. B 79 (2009) 220512.

[8] H. Ogino, Y. Shimizu, K. Ushiyama, N. Kawaguchi, K. Kishio, J. Shimoyama, Appl. Phys. Express 3 (2010) 063103.

[9] H. Ogino, S. Sato, K. Kishio, J. Shimoyama, T. Tohei, Y. Ikuhara, Appl. Phys. Lett. 97 (2010) 072506.

[10] J. Shimoyama, H. Ogino, A. Yamamoto, K. Kishio, Solid State Commun. 152 (2012) 640.

[11] M. Tropeano, I. Pallecchi, M. R. Cimberle, C. Ferdeghini, G. Lamura, M. Vignolo, A. Martinelli, A. Palenzona, M. Putti, Supercond. Sci. Technol. 23 (2010) 054001.

[12] A. S. Sefat, S. Michael, A. McGuire, B. C. Sales, R. Jin, J. Y. Howe, D. Mandrus, Phys. Rev. B 77 (2008) 174503.

[13] S. J. Singh, J. Prakash, A. K. Ganguli, S. Patnaik, IEEE Trans. Appl. Supercond. 21 (2011) 
2870.

[14] L. Pinsard-Gaudart, D. Berardan, J. Bobroff, N. Dragoe, Phys. Stat. Sol. 2 (2008) 185.

[15] H. Kitagawa, H. Noguchi, T. Kiyabu, M. Itoh, Y. Noda, J. Phys. Chem. Solids 65 (2004) 1223.

[16] B. Lenoir, A. Dauscher, M. Cassart, Y. I. Ravich, H. Scherer, J. Phys. Chem. Solids 59 (1998) 129.

[17] H. Hosono, K. Tanabe, E. Takayama-Muromachi, H. Kageyama, S. Yamanaka, H. Kumakura, M. Nohara, H. Hiramatsu, S. Fujitsu, Sci. Technol. Adv. Mater. 16 (2015) 033503.

[18] H. J. Goldsmid, Proc. Phys. Soc. London 71 (1958) 633.

[19] I. Tarasaki, Y. Sasago, K. Uchinokura, Phys. Rev. B 56 (1997) R12685.

[20] D. Y. Chung, T. Hogan, P. Brazis, M. R. Lane, C. Kannewurf, M. Bastea, C. Uher, M. G. Kanatzidis, Scienece 287 (2000) 1024.

[21] A. Omachi, J. Kajitani, T. Hiroi, O. Miura, Y. Mizuguchi, J. Appl. Phys. 115 (2014) 083909.

[22] S. J. Singh, H. Ogino, J. Shimoyama, K. Kishio, Supercond. Sci. Technol. 26 (2013) 105020.

[23] D. J. Singh, Phys. Rev. B 78 (2008) 094511.

[24] I. I. Mazin, J. Schmalian, Physica C 469 (2009) 614.

[25] M. Tropeano, A. Martinelli, A. Palenzona, E. Bellingeri, E. G. d'Agliano, T. D. Nguyen, M. Affronte, M. Putti, Phys. Rev. B 78 (2009) 094518.

[26] Y. Machida, K. Tomokuni, T. Isono, Y. Nakajima,T. Tamegai, J. Phys. Soc. Japn. 78 (2009) 073705. 
[27] Z. J. Xiang, X. G. Luo, J. J. Ying, X. F. Wang, Y. J. Yan, A. F. Wang, P. Cheng, G. J. Ye, X. H. Chen, Phys. Rev. B 85 (2012) 224527.

[28] H. Yang, Y. Liu, C. Zhuang, J. Shi, Y. Yao, S. Massidda, M. Monni, Y. Jia, X. Xi, Q. Li, Z.-K. Liu, Q. Feng, H.-H. Wen, Phys. Rev. Lett. 101 (2008) 067001.

[29] F. Han et al., Sci. China. Ser. G 53 (2010) 1202.

[30] G. D. Mahan, B. Sales, J. Sharp, Physics Today 50(3) (1997) 42. 


\section{Figure Captions:}

Fig. 1. Crystal structure of Sm1111, $\left(\mathrm{Fe}_{2} \mathrm{As}_{2}\right)\left(\mathrm{Ca}_{4}\left(\mathrm{Mg}_{0.25} \mathrm{Ti}_{0.75}\right)_{3} \mathrm{O}_{8}\right)$ (22438) and $\left(\mathrm{Fe}_{2} \mathrm{As}_{2}\right)\left(\mathrm{Ca}_{5}\left(\mathrm{Sc}_{0.5} \mathrm{Ti}_{0.5}\right)_{4} \mathrm{O}_{11}\right)(2254 \underline{11})$.

Fig. 2. Temperature dependence on (a) the resistivity $(\rho)$ (b) thermoelectric power $(S)$ (c) thermal conductivity $(\kappa)$ for samples Sm1111, 22438 and $2254 \underline{11}$ and (d) the temperature dependence of $\kappa$ in zero and $9 \mathrm{~T}$ magnetic field below $100 \mathrm{~K}$.

Fig. 3. The dependence of maximum thermoelectric power $\left(|S|_{\max }\right)$ with respect to (a) nominal content of fluorine $(x)$ and (b) transition temperature $\left(T_{\mathrm{c}}\right)$.

Fig. 4. The variation of Hall coefficient $\left(R_{\mathrm{H}}\right)$ with respect to temperature for 22438,225411 and $\mathrm{SmFeAsO}_{0.88} \mathrm{~F}_{0.12}$ and $\mathrm{SmFeAsO}_{0.8} \mathrm{~F}_{0.2}$. The inset shows the field dependence of Hall resistivity $\left(\rho_{\mathrm{xy}}\right)$ for 22438 at various temperatures.

Fig. 5. Dimensionless figure of merit $(Z T)$ as a function of temperature for $22438,2254 \underline{11}$, $\mathrm{SmFeAsO}_{0.88} \mathrm{~F}_{0.12}$ and $\mathrm{SmFeAsO}_{0.8} \mathrm{~F}_{0.2}$ samples. 
Fig. 1

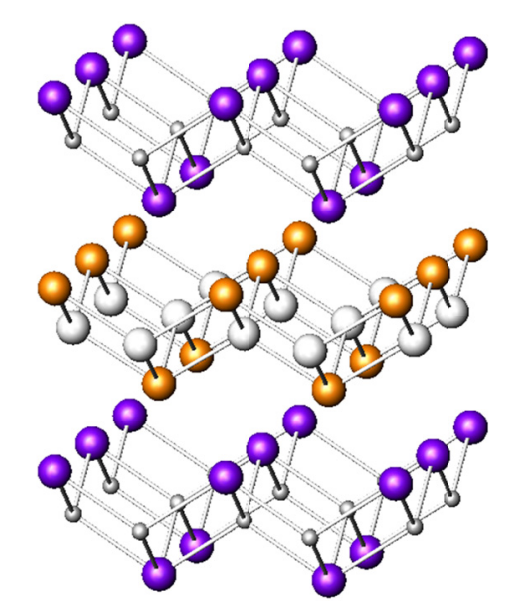

1111
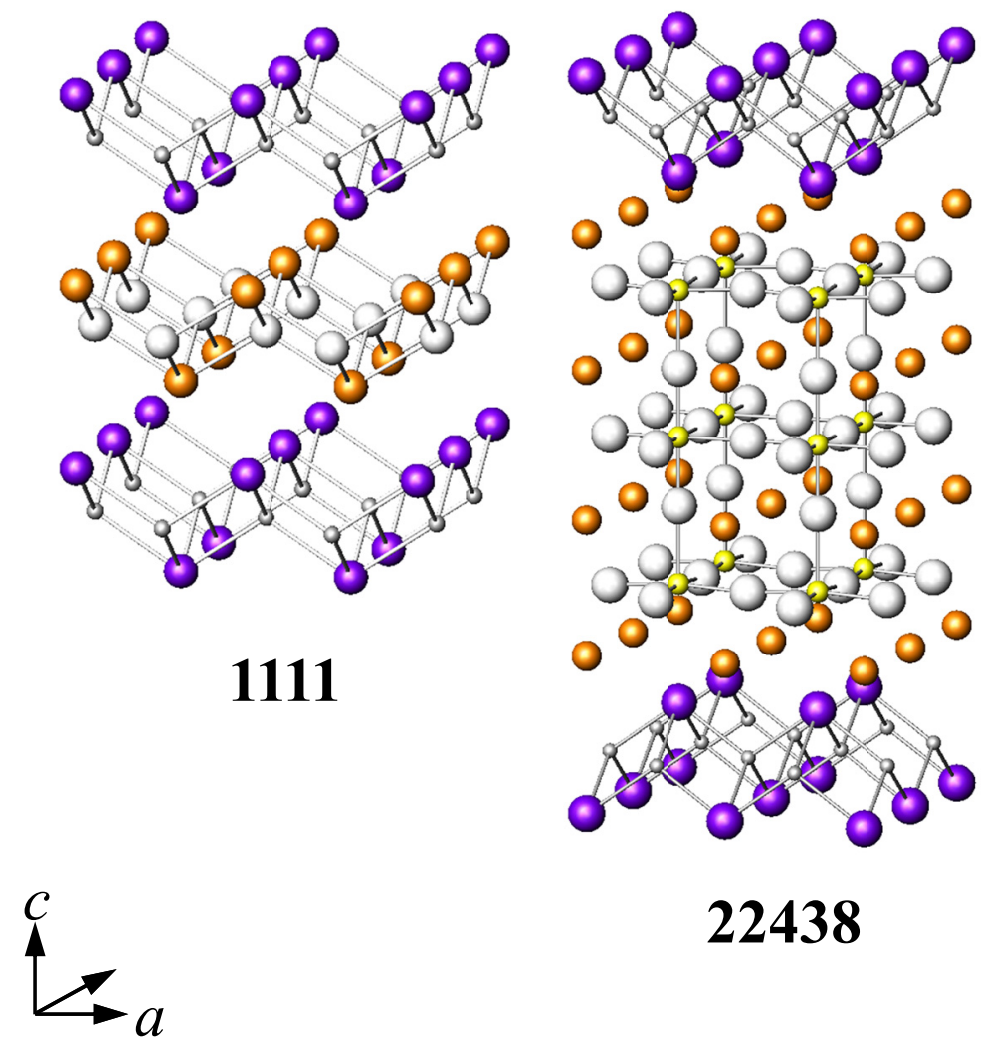

22438

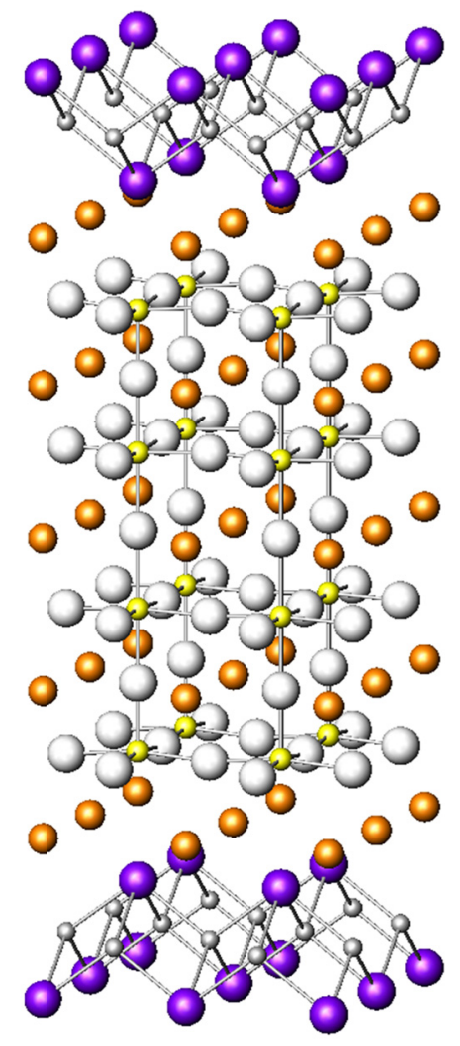

225411 
Fig. 2

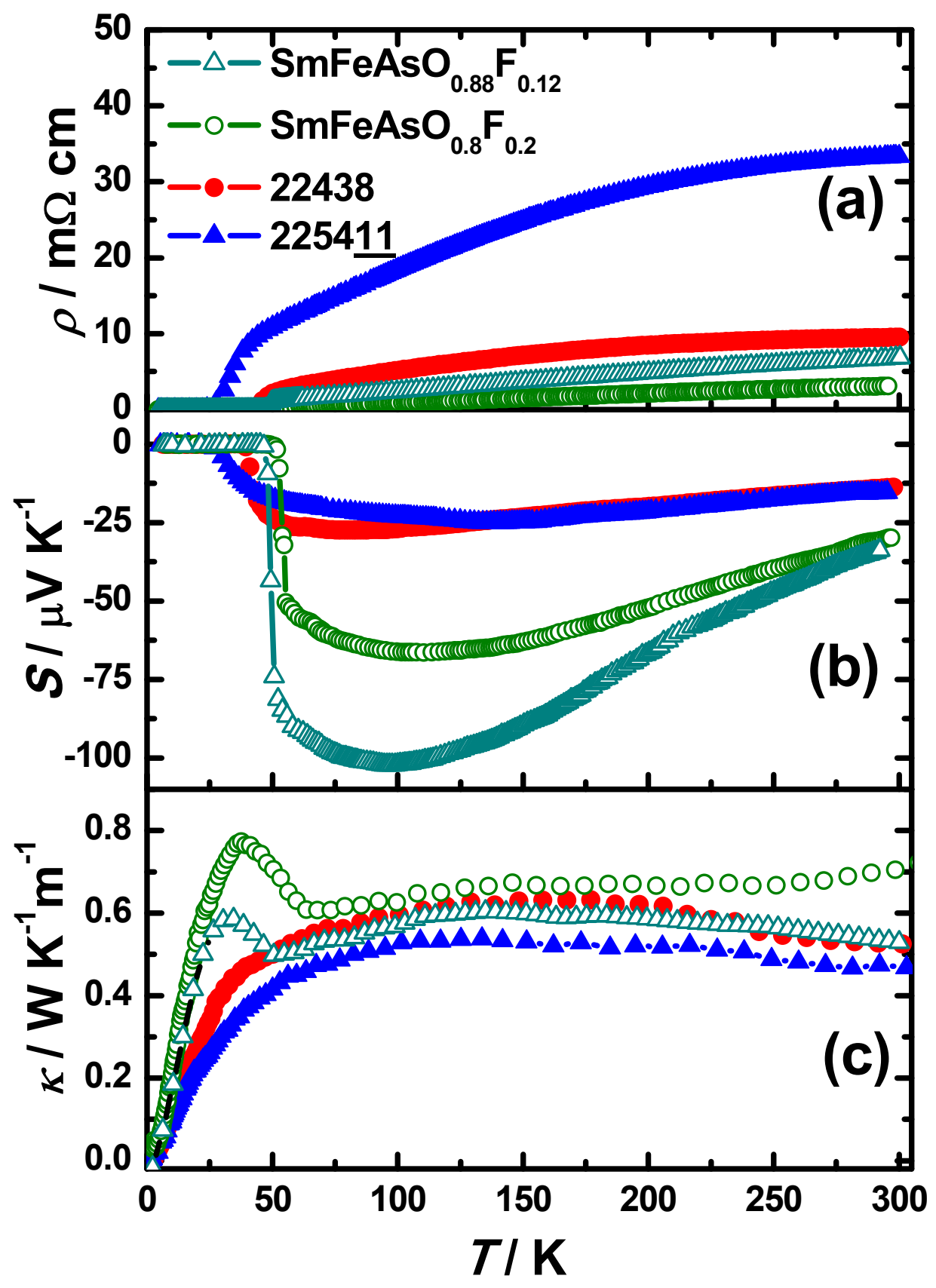




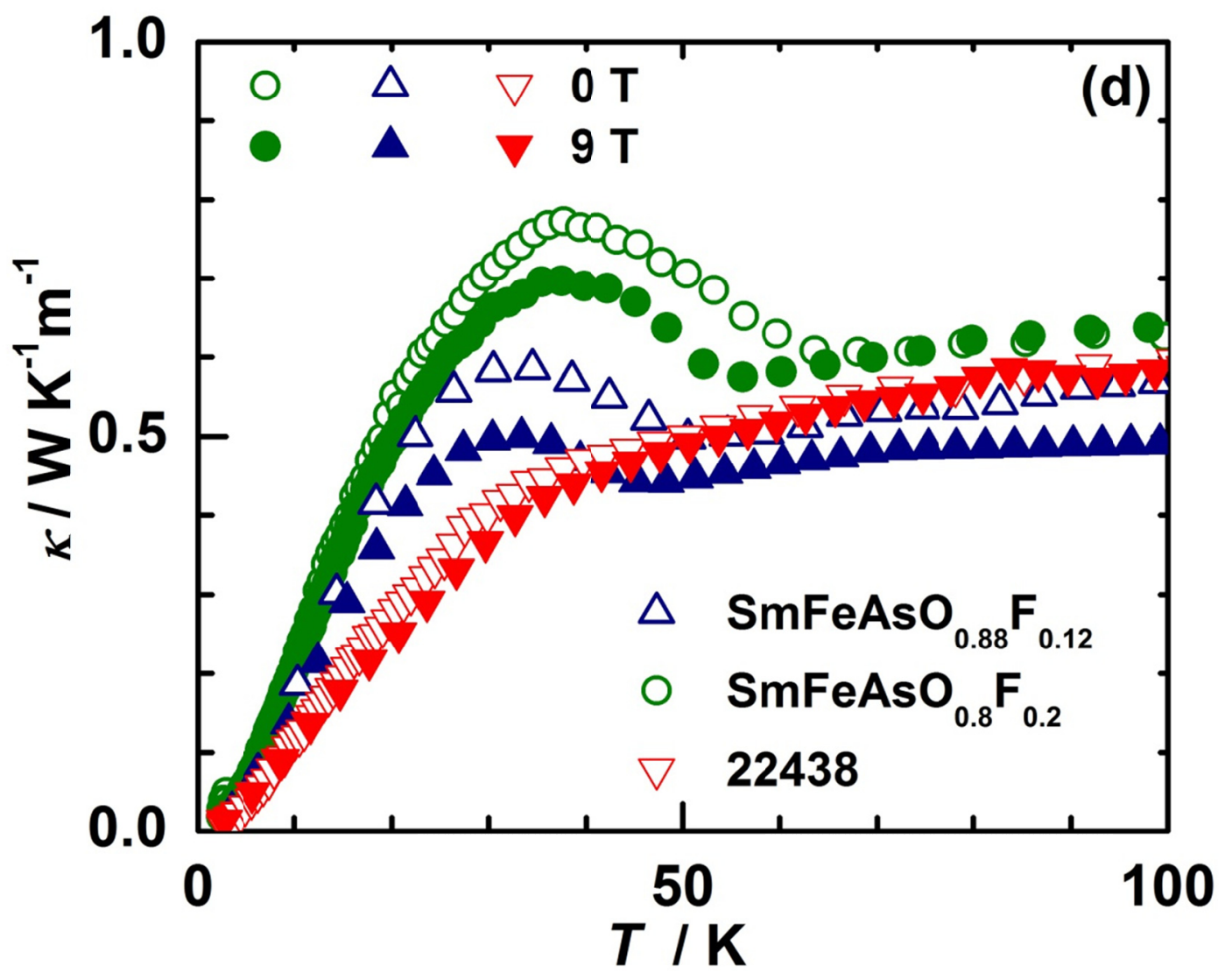


Fig. 3
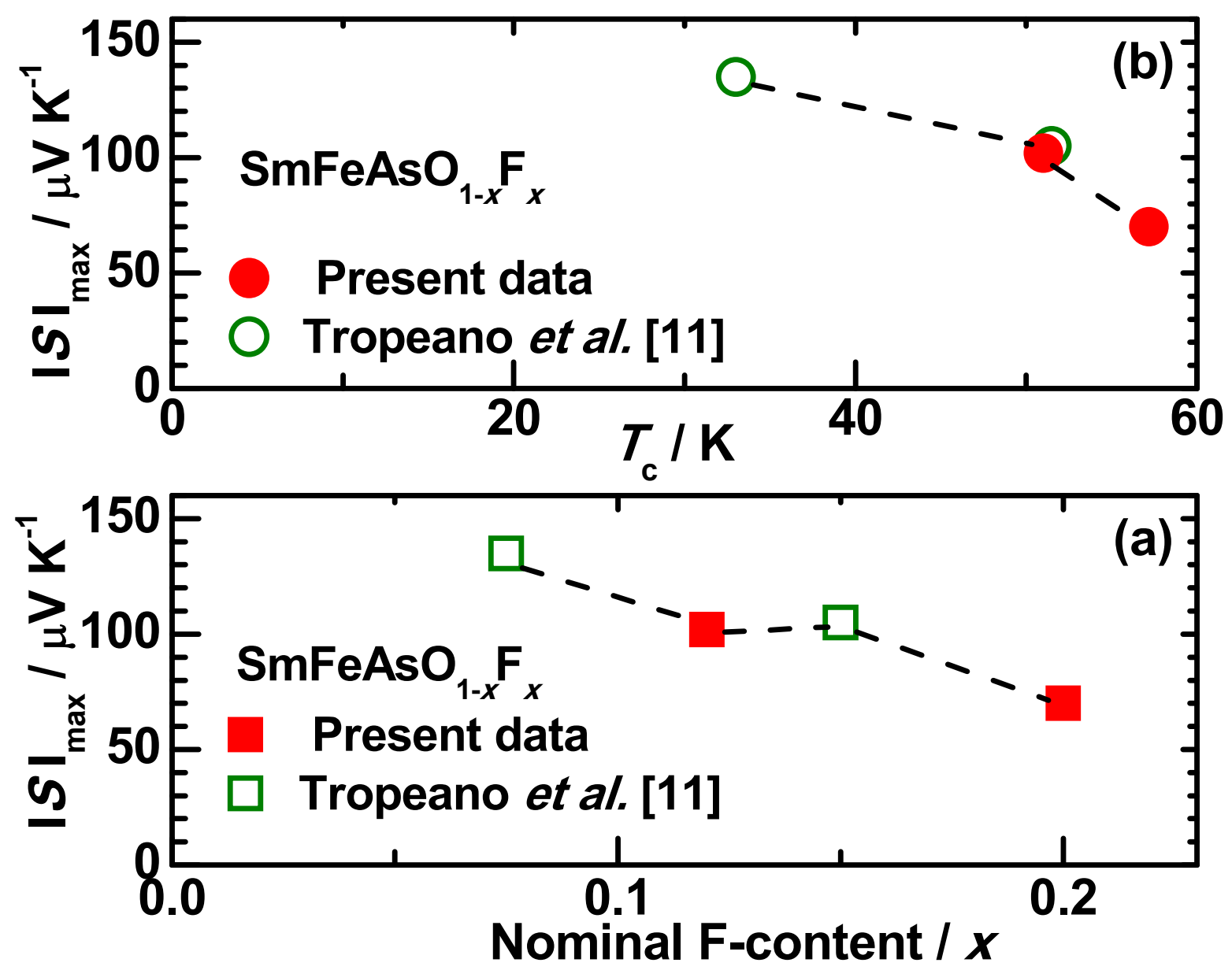
Fig. 4

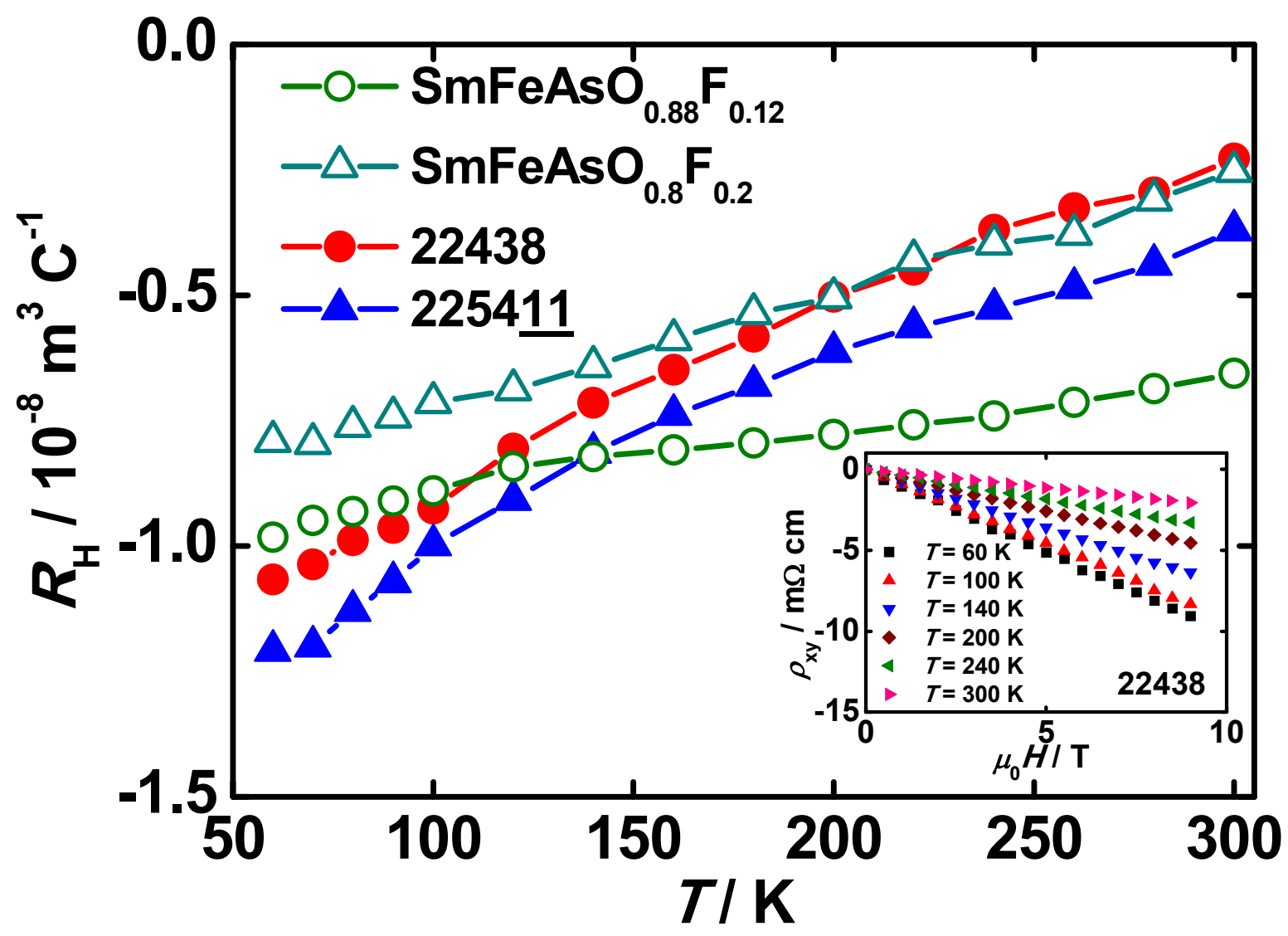


Fig. 5

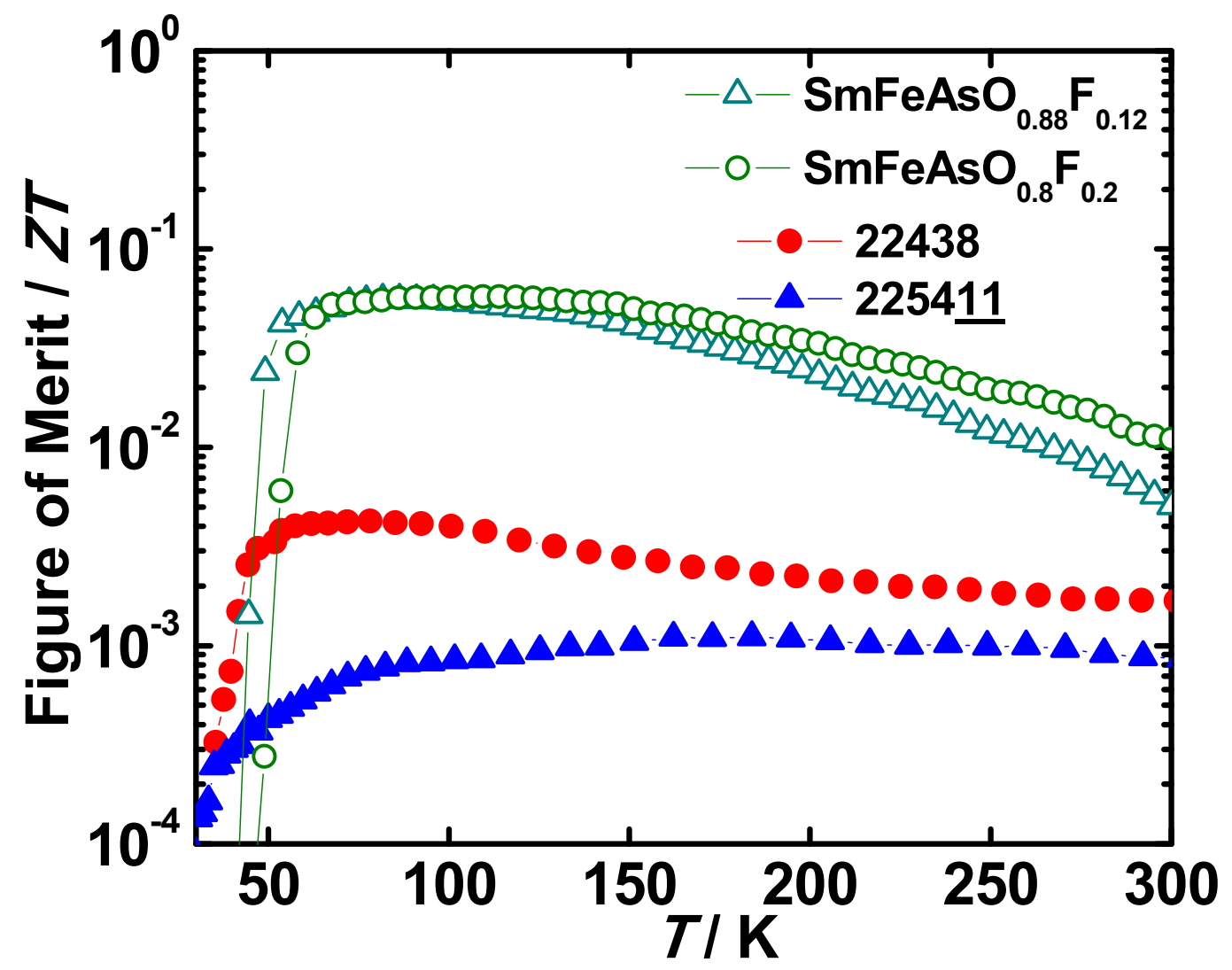

